\title{
A Note on Mountford's manuscript "Can a Brain Drain be good for growth in the source economy?"
}

\author{
Pierpaolo Giannoccolo*
}

$10 / 11 / 2008$

\begin{abstract}
In this briefly note we extend the Mountford's article "Can a Brain Drain be good for growth in the source economy?" by introducing an active role of the government in the education decisions of the agent by public education, taxes and subsidies. First, we demonstrate that the main results obtained by Mountford do not disappeared. Second, we argue that the model, by these new assumptions, help us to extend the Mountford's results to a greater range of economic scenarios and in particular, to the new enlarged European Union.
\end{abstract}

JEL classification: H52; O15

Keywords:

Brain Drain, Public Goods, Education, Taxes.

*University Alma Mater of Bologna. Department of Economics pierpaolo.giannoccolo@unibo.it 


\section{Introduction}

In this note we propose an extension of the article "Can a Brain Drain be good for growth in the source economy?" by Mountford (1997). Differently from the standard "Brain Drain" literature, the Mountford's model identifies some conditions in which the incentive to acquire skills may be strengthened by the possibility to migrate. In particular, he shows that when migration is not a certainty, a Brain Drain (BD hereafter) may increase the average education level of those left behind in the source economy. These results are also argued most recently by Stark and Wang (2002), Stark et al. (1997) and Stark (2004) and this new field of the BD literature is called "Brain Gain". The main mechanism of the Mountford's model is that when region $J$ is more productive than region $I$ and the probability to migrate, $\pi$, is low, then BD may increase average productivity and equality in the source economy. In particular, the two key assumptions of the model: high differentials on the productivity between the sending and the receiving country and absence of public education (no government role is analyzed in Mountford) bound the result to Less Developed Countries where typically there are not sufficient resources to invest in public education and which suffer a drain of high skilled workers to High Developed Countries.

Aim of this note is to extend the Mountford's model in two different directions - by introducing public education and taxation/subsidies - in order to demonstrate that these new specifications help us to extend the analysis without loose the main results of the original model. Furthermore, this new version can be useful to analyze the new European Union (EU hereafter) scenario where it is possible to identify the existence of the two key factors that could drive the BD in a Brain Gain. From one side, we can distinguish two different "clubs" of members: the former, with higher productivity and growth, and the new members with lower productivity ${ }^{1}$. From the other side, during the first period of this enlargement the real opportunity to migrate in the former EU countries for high skilled workers of new members ( $\pi$ in Mountford) it is not very high ${ }^{2}$. To complete the analysis and test the possibility of Brain Gain, we need to

\footnotetext{
${ }^{1}$ Labour costs in 2006 varied by one to twenty in the EU27. Expressed in euro, the average hourly labour cost3 in the EU27 in 2006 was $€ 20.35$. Sweden $(€ 32.16)$ had the highest hourly labour cost in 2006, followed by Denmark (€31.98 in 2005), Luxembourg (€31.98) and Belgium (€31.58). Bulgaria (€1.65), Romania (€2.68), Latvia (€3.41) and Lithuania (€4.21) had the lowest. Within the structure of the labour costs, the highest share of social security costs paid by the employer was found in Sweden (30.6\%), followed by Belgium (30.3\%) and France $(28.6 \%)$ and the lowest shares in Malta (6.9\%), Denmark (10.9\%), Slovenia $(13.4 \%)$ and Cyprus (15.1\%). (Eurostat Yearbook 2008).

${ }^{2}$ Analyzing the migration policies of the EU we identify a dual approach: a) internal migration of EU nationals has been harmonized at the EU level, becoming essentially free by 2014 for the whole EU27. b) External migration rules are not harmonized as they generally fall within the responsibility of each individual member state. Furthermore, from 2006 policies relating to the free movement of workers of the EU-15 states could be classified into three categories: i) Keeping the restrictions in place for at least three more years: Austria, Germany; ii) Lifting the restrictions gradually, within the next three years: Belgium, Denmark, France, Luxembourg, the Netherlands; iii) Keeping labor markets open/removing restrictions: Finland, Greece, Ireland, Portugal, Spain, Sweden, Italy, United Kingdom; All countries except Finland have opted for transition periods for workers from Bulgaria and Romania.
} 
extend the theoretical framework proposed by Mountford. First we introduce a government's role in the educational decisions of agents thought the introduction of educational subsidies. The main point is the stylized fact that education is predominantly a public good in the $\mathrm{EU}^{3}$. Second we introduce the presence of taxation for educated agents and subsidies to uneducated ones in order to capture from one side the redistributive role of the EU governments and from the other side the necessity for the government to compensate the expenses on public education.

The note is organized as follows. There are two sections which follow the introductory section: the Autarchic case and the Mobility case) first section of the Mountford's article. First, we defines the main assumptions and the structure of the models. Second, we introduce the government by public education, subsidies and taxes. Third, we analyze the new model and we compare our results with the Mountford's ones. Finally we concludes with recommendations for further extensions in the future.

\section{Autarchic case}

Following the Mountford's framework, we propose a model that analyzes a small open overlapping generations economy with one good and perfect capital mobility. The world's one good is produced under constant returns to scale by two factors, capital and efficiency units of labor. There are a continuum of agents in each generation (normalized to unity) and there is not population growth. Agents chose if be educated or uneducated.

Mountford shows how a BD can be beneficial to the productivity level of the source economy when there is a "growth externality" associated with the proportion of educated workers in the economy in the previous period.

Let us resume the main assumptions of the Mountford's model ${ }^{4}$. Individual possess different levels of latent ability $\left(e^{i}\right)$ distributed over the closed interval $[0, E]$, according to the density function $g\left(e^{i}\right)$, where, by definition, $\int_{0}^{E} g e^{i} d e^{i}=1 ; g\left(e^{i}\right)>0 \forall e^{i} \in[0, E]$. Agents exist in an overlapping generations world and live for three periods. In the first periods can invest resources in education, resources that they borrow from the capital market at the world's rate of interest $r^{*}$. The cost of education is assumed fixed at $c$ units of output. Agents educated obtain $e^{i}$ efficiency units of labor in the second period of their life and uneducated have only one efficiency unit of labor. Agents can only work in the second period and in this period the agent must repay the debt of the first period and save in order to consume in the third and last period of life. All

\footnotetext{
${ }^{3}$ In Austria, Denmark, Finland, Greece and Poland the totality of the tertiary education is provided by government Moreover in Belgium, Czech Republic, France, Germany, Ireland Italy, Portugal, Slovak Republic and Sweden we have al least the $80 \%$ of tertiary education publicly financed. Finally, In Hungary, Netherlands, Spain and United Kingdom the share is from 70 to 80 percent (OECD 2004).

${ }^{4}$ See chapter 2 of Mountfors (1997) for furthermore details on production of goods, factor prices, education decision and growth externality.
} 
agents have the same preferences and access to the same technology but different levels of latent ability. The optimal decision for agent $i$ will be to invest in education if $\lambda_{t} w(k) e^{i}>\lambda_{t} w(k)+c\left(1+r^{*}\right)$ where $w(k) \equiv\left[f(k)-k f^{\prime}(k)\right]$ is the wage rate per efficiency unit of labor and $\lambda_{t}$ is the level of technology. Thus all agents with a latent ability greater than $e^{*}$ is uniquely defined by the following equality:

$$
e^{*}=\frac{\lambda_{t} w(k)+c\left(1+r^{*}\right)}{\lambda_{t} w(k)} \equiv e^{M} .
$$

Let us now define $e^{M}$ the value of $e^{*}$ identified by Mountford in the no migration case (eq. 7, pg. 291). In our paper, this is the value of $e^{*}$ in which there is no migration and there is not a government role on education decision.

Furthermore, let us introduce new hypotheses to the Mountford's model in order to extend his results. Let us assume that there exist a government which taxes educated workers, subsidizes educational costs and has a redistributive role. The timing of the model does not change in the first and third period. Although, in the second period, educated agents pay a lump sum tax $T$ and receive a subsidy of $\gamma c\left(1+r^{*}\right)$ where $\gamma \in[0,1]$ and uneducated agents receive $b$. Following these new assumptions, in autarchic case and with taxation/subsidies scheme, $e^{*}$, is uniquely defined by the following equality:

$$
e_{A, T}^{*}=e^{M}+\frac{b+T-\gamma c\left(1+r^{*}\right)}{\lambda_{t} w(k)} .
$$

Let us introduce a Budget Constraint for the government

$$
s_{t} T-s_{t} \gamma c-\left(1-s_{t}\right) b=0,
$$

where $s_{t}=\int_{e_{A, T}^{*}}^{E} g e^{i} d e^{i}$ is the amount of educated agents. When, as in Mountford, we consider the case of uniformly distributed abilities (i.e. $g\left(e^{i}\right)=\frac{1}{E}$ ), equation 3 becomes

$$
\left(1-\frac{e^{*}}{E}\right)(T-\gamma c)-\frac{e^{*}}{E} b=0 .
$$

Furthermore, let us assume that the government maximizes the utility of the worst-off workers, by using a Maximin criterion. By definition, this workers is the uneducated and

$U\left(\lambda_{t} w(k)+b\right)=U\left(\lambda_{t} w(k) \lambda_{t} w(k) e_{A, T}^{*}-T-(1-\gamma) c\left(1+r^{*}\right)\right)$.

The maximization program of the government is

$$
\begin{gathered}
\underset{b, T}{\operatorname{Max}} U\left(\lambda_{t} w(k)+b\right) \\
\text { s.to } \quad s_{t} T \geq s_{t} \gamma c+\left(1-s_{t}\right) b
\end{gathered}
$$

or, equivalently

$$
\underset{T}{\operatorname{Max} b}
$$


where, by equations (2) and (3)

$$
\begin{gathered}
b=-\frac{1}{2}\left(\lambda_{t} w(k) e^{M}+2 T-2 \gamma c-\gamma c r^{*}\right)+ \\
+\frac{1}{2}\left[\begin{array}{c}
\left(\lambda_{t} w(k) e^{M}+2 T-2 \gamma c-\gamma c r^{*}\right)^{2}+ \\
+4\left(\lambda_{t} w(k)\left(E-e^{M}\right)-T+\gamma c\left(1+r^{*}\right)\right)(T-\gamma c)
\end{array}\right]^{\frac{1}{2}} .
\end{gathered}
$$

Solving equation (6), the optimal value of taxation is

$$
T^{*}=\frac{\lambda_{t} w(k)\left(E^{2}-\left(e^{M}\right)^{2}\right)}{4 E}+\gamma c+\left(\frac{e^{M} r^{*}}{2 E}\right) \gamma c-\frac{\left(\gamma c r^{*}\right)^{2}}{4 \lambda_{t} w(k) E}>0
$$

and $e^{*}$, becomes

$$
e_{A, T}^{*}=e^{M}+\lambda_{t} w(k)\left(E^{2}-\left(e^{M}\right)^{2}\right)+2\left(e^{M} r^{*}\right) \gamma c-\frac{\left(\gamma c r^{*}\right)^{2}}{\lambda_{t} w(k)}>e^{M} .
$$

These results are resumed in the following propositions.

Proposition 1 Consider the economy described above. There exist a positive level of optimal taxation which is compatible with a redistributive role of a government and public subsidies to education costs.

Proof. See Appendix.

Proposition 2 The presence of governments implies an higher amount of educated agents respect the case without government analyzed in Mountford.

Corollary 3 Higher is $e^{M}$, lower is the gap between $e_{A, G}^{*}$ and $e^{M}$ and higher are the subsidies and the educational costs, higher are this gap.

Proof. See Appendix.

The first proposition implies that, starting from the theoretical framework proposed by Mountford in the autarchic case, it is possible introduce a government which subsidizes the education and realizes a redistributive policy by implementing a maximization program. Furthermore, the second proposition give us a comparison between the Mountford's results and the model with public education and taxes. In the autarchic case, the introduction of a government implies an increase in the amount of educated. Furthermore (Corollary 3 ) the gap between the amount of educated workers in the case without and with government is negatively correlated to $e^{M}$ and positively correlated to the amount of subsidies and educational costs.

Finally, as in Mountford, we assume that there is an economy wide growth externality related to the proportion of educated workers in the economy in the 
previous period. Thus $\lambda_{t}=\lambda\left(s_{t-1}\right), \lambda^{\prime}\left(s_{t-1}\right)>0, \lambda(0)=1$, and $\lambda(1)$ is finite. Also in this extended model the only dynamics depend on from the growth externality and the proportion of educated workers in period $t$ is an increasing function of educated workers in $t-1$.

Analyzing the dynamics of the model, we derive the following proposition.

Proposition 4 When we introduce the government, there exist at least one steady state equilibrium for $s_{t}$ as in the Mountford case.

Proof. See Appendix.

This fourth proposition specifies that the introduction of public education, taxes and subsidies does not change the dynamics identified in the model of Mountford and consequently we have at least one steady state equilibrium.

\section{Mobility case}

In this section we introduce migration (only for educated agents) ${ }^{5}$. As in Mountford, we examine the case where emigration is limited by the receiving country i.e. immigration controls - the probability of successful emigration $\pi$ is independent of the number of workers who are eligible to migrate, and the emigration policy is fully anticipated. Thus all agents with a latent ability greater than $e^{*}$ is uniquely defined by the following equality:

$$
e^{*}=\frac{\lambda_{t} w(k)+c\left(1+r^{*}\right)}{\pi w^{F}+(1-\pi) \lambda_{t} w(k)} \equiv e_{\pi}^{M}
$$

where $w^{F}$ is the wage per efficiency unit of labor in the world economy (by assumption it is always higher than in the small open economy $\left.\lambda_{t} w(k)\right)$. We define $e_{\pi}^{M}$ the value of $e^{*}$ identified by Mountford in the migration case (eq. 14, pg. 294). In our paper, this is the value of $e^{*}$ in which there is migration and there is not a government role on education decision. When we introduce a government $e^{*}$ becomes

$$
e_{\pi, G}^{*}=e_{\pi}^{M}+\frac{b+(1-\pi) T-\gamma c\left(1+r^{*}\right)}{\bar{w}}
$$

where $\bar{w}=\pi w^{F}+(1-\pi) \lambda_{t} w(k)>\lambda_{t} w(k)$.

The Budget Constraint is,

$$
\left(1-\frac{e^{*}}{E}\right)((1-\pi) T-\gamma c)-\frac{e^{*}}{E} b=0 .
$$

The maximization program of the government is

\footnotetext{
${ }^{5}$ See paragraph 2.2 of Mounftford (1997) for furthermore details on the migration hypothesis and the analysis of the general emigration. We focus the attention on the BD case (paragraph 2.2.1.2).
} 


$$
\begin{gathered}
\underset{b, T}{\operatorname{Max}} U\left(\lambda_{t} w(k)+b\right) \\
\text { s.to }(1-\pi) s_{t} T \geq s_{t} \gamma c+\left(1-s_{t}\right) b
\end{gathered}
$$

or, equivalently

$$
\underset{T}{\operatorname{Max}} b
$$

where, by equations (9) and (10)

$$
\begin{gathered}
b=-\frac{\left(\bar{w} e_{\pi}^{M}+2(1-\pi) T-2 \gamma c-\gamma c r^{*}\right)}{2}+ \\
+\frac{1}{2}\left[\begin{array}{c}
\left(\bar{w} e_{\pi}^{M}+2(1-\pi) T-2 \gamma c-\gamma c r^{*}\right)^{2}+ \\
+4\left(\bar{w}\left(E-e_{\pi}^{M}\right)-(1-\pi) T+\gamma c\left(1+r^{*}\right)\right)((1-\pi) T-\gamma c)
\end{array}\right]^{\frac{1}{2}} .
\end{gathered}
$$

Solving equation (11), the optimal value of taxation is

$$
T^{* *}=\frac{\bar{w}\left(E^{2}-\left(e_{\pi}^{M}\right)^{2}\right)}{4 E(1-\pi)}+\frac{\gamma c}{(1-\pi)}+\left(\frac{e_{\pi}^{M} r^{*}}{2(1-\pi) E}\right) \gamma c-\frac{\left(\gamma c r^{*}\right)^{2}}{4(1-\pi) \bar{w} E}>T^{*}
$$

and $e_{\pi, G}^{*}$, becomes

$$
e_{\pi, G}^{*}=e_{\pi}^{M}+\bar{w}\left(E^{2}-\left(e_{\pi}^{M}\right)^{2}\right)+2\left(e_{\pi}^{M} r^{*}\right) \gamma c-\frac{\left(\gamma c r^{*}\right)^{2}}{\bar{w}} .
$$

These results are resumed in the following propositions.

Proposition 5 Consider the economy described above. In presence of migration, there exist a positive level of optimal taxation which is compatible with a redistributive role of a government and public subsidies to education costs.

Proof. See Appendix.

Proposition 6 The presence of governments implies an higher amount of educated agents respect the case without government analyzed in Mountford.

Corollary 7 Higher is $e^{M}$, lower is the gap between $e_{A, G}^{*}$ and $e^{M}$ and higher are the subsidies and the educational costs, higher are this gap.

Corollary 8 The migration implies an higher level of taxation respect the autarchic case.

Proof. See Appendix.

Correspondingly the first proposition, the fifth proposition implies that, starting from the theoretical framework proposed by Mountford in the mobility 
case, it is possible introduce a government which subsidizes the education and realizes a redistributive policy by implementing a maximization program. Furthermore, the sixth proposition give us a comparison between the Mountford's results and the model with public education and taxes in presence of mobility. Also comparing the mobility case of both version, the introduction of a government implies an increase in the amount of educated. Furthermore (by Corollary 6) the gap between the amount of educated workers in the case without and with government is negatively correlated to $e_{\pi}^{M}$ and positively correlated to the amount of subsidies and educational costs. Finally (by Corollary 7 ) according to the economic intuition, the migration implies an higher level of taxation respect the autarchic case in order to compensate the decrease of the contributive basis.

As in Mountford, we study the case in which the source economy can benefit from a Brain Gain. The result of this analysis is summarized by the following proposition.

Proposition 9 Consider the economy described above. In presence of migration and a government. There will exist a positive optimal level of "Brain Drain" emigration if

$$
g\left(e_{A, T}^{*}\right)\left(\begin{array}{c}
\left(\lambda_{t} w(k)+c\left(1+r^{*}\right)\right) * \\
*\left(1-2 \lambda_{t} w(k)-2 c\left(1+(1-\gamma) r^{*}\right)\right)+ \\
-\left(E^{2}-\left(e_{\pi}^{M}\right)^{2}\right)\left(\lambda_{t} w(k)\right)^{2}-\left(\gamma c r^{*}\right)^{2}
\end{array}\right) \frac{\left(w^{F}-\lambda_{t} w(k)\right)}{\left(\lambda_{t} w(k)\right)^{2}}>\frac{1}{4} .
$$

Proof. See Appendix.

As in Mountford, the source economy can benefit from a brain drain so long as there are a sufficient number of people who decide to be educated. Analyzing the effect of the government introduction in the Mountford model, we derive the following proposition.

Proposition 10 The presence of governments implies that the conditions to obtain brain gain are more strict respect the case without government analyzed in Mountford.

Proof. See Appendix.

The ninth proposition implies that the introduction of the government in the model of Mountford does not prevent the existence of a positive optimal level of BD (and consequently a Brain Gain). Furthermore, the tenth proposition give us a comparison between the Mountford's results and the model with public education and taxes. In this second case, the conditions to obtain a Brain Gain are more strict and consequently the probability that the migration of educated workers implies a BD for the sending country increases. 


\section{Conclusion}

In this briefly note we demonstrate that by introducing an active role of the government in the education decisions of the agent by public education, taxes and subsidies, we do not change the main results that Mountford obtain in the first section of his work. Furthermore, these new assumptions allow us to extend these results to different scenario as the enlarged European Union. In this context this new specification of the model of Mountford can be a basis to recent economic and political debates in Europe.

First, by introducing public education we can use the model to contribute to the debate on the amount of investment in education in Europe. For example, the possibility to $\mathrm{BD}$ from the new EU regions to the former ones and the massive $\mathrm{BD}$ from the $\mathrm{EU}$ to the USA implies that the EU countries (formers and new ones) reduce the investment in education. In this scenario the optimal policies of the EU institutions is to coordinate all the regions and to define a target in the investment in education and research. This coordination is the target of the "Lisbon Strategy". Furthermore, the subsequent Spring European Councils (Lisbon 2000, Stockholm 2001, Barcelona 2002 and Brussels in 2003) have endorsed the ERA (European Research Area) and set a series of objectives inviting the Commission and the Member States to take due account of the possible shortage of human resources in $\mathrm{R} \& \mathrm{D}$ as well as of the importance of enhancing the training and mobility of researchers.

Secondly, this model can be used inside the recent debate on the policies to reverse the European Brain Drain. Furthermore, it is important to note that the EU performs a crucial role in these policies by collecting and reviewing the existent policies and by acting as coordinator of common policies inside the EU. More deeply, first the EU commission by several Councils and Reports reminds that it is not sufficient increase the investment in Research but we have to stop the European Brain Drain, we have to reverse it, "Europeans who have moved abroad would love to come home" and we have to remember that the "Brain Drain should work in both directions" by attracting foreign brilliant scientists. Moreover, the European Commission trays to coordinate the BD policies of the Member countries. In particular, it suggests, from one side, to invest more in research (3\% of GDP) and, from the other side, to implement policies and strategies to reverse the Brain Drain and "make the Europe attractive to the researchers from the rest of the world".

Finally, this model can be used to analyze a new scenario where the former EU regions have not high differences in the productivity and they compete by attracting educated workers (migration competitions) from the new entrant. In recent years, there are several "Brain Drain Competition" policies implemented in Europe. The key strategies and mechanisms used to attract foreign research graduates in Europe are: to make the academic system more open and flexible; to improve the regulatory conditions particularly on immigration; to improve the sign-posting and information at national level; to dedicate grants for for-

eign researchers; to adapt income situations to market forces; to provide tax reductions specifically for researchers and knowledge workers; more active in- 
ternational marketing and support for international researchers. It is possible to divide these policies in seven macro-groups: immigration policies, incentives to the researchers and their family, grants and scholarships, tax and salaries, investment in Research, marketing and recruiting policies, studies and analysis of the immigration policies of the others countries.

\section{References}

[1] Eurostat (2008). Eurostat statistical yearbook 2008 - Europe in figures - published by the Statistical Office of the European Communities.

[2] Mountford, A. (1997) Can a brain drain be good for growth in the source economy? Journal of Development Economics, 53.

[3] OECD (2004) Education at a Glance. 2004 edition.

[4] Stark O. (2004). Rethinking the Brain Drain. World Development, 32, 1 $15-22$.

[5] Stark O. et al. (1997). A Brain Gain with Brain Drain. Economics Letters, $55,227-234$.

[6] Stark O. and Y. Wang (2002). Inducing human capital formation: migration as a substitute for subsidies. Journal of Public Economics, 86, 1. 


\section{Appendix A - proofs}

Proof of Proposition (1): $T^{*}$ is always positive hence $e^{M}\left(\frac{r^{*} \gamma c}{2 E}\right)=\left(\frac{r^{*} \gamma c}{2 E}\right)+$ $\left(\frac{c}{\lambda_{t} w(k)}\right)\left(\frac{r^{*} \gamma c}{2 E}\right)+\left(\frac{\gamma\left(c r^{*}\right)^{2}}{2 \lambda_{t} w(k) E}\right)>\left(\frac{\gamma\left(c r^{*}\right)^{2}}{2 \lambda_{t} w(k) E}\right)>\frac{\left(\gamma c r^{*}\right)^{2}}{4 \lambda_{t} w(k) E}$ by the assumption $\gamma \leq$ 1 , then $\left(\frac{e^{M} r^{*}}{2 E}\right) \gamma c-\frac{\left(\gamma c r^{*}\right)^{2}}{4 \lambda_{t} w(k) E}>0$ and by $E>e^{M}$ then $\frac{\lambda_{t} w(k)\left(E^{2}-\left(e^{M}\right)^{2}\right)}{4 E}>0$. Furthermore, the equation (2) and the maximin criterion ensure level of taxes lower enough to have educated agents.

Proof of Proposition (2): By $E>e^{M}$ and $2 e^{M}\left(r^{*} \gamma c\right)=2\left(r^{*} \gamma c\right)+$ $2\left(\frac{c}{\lambda_{t} w(k)}\right)\left(r^{*} \gamma c\right)+2\left(\frac{\gamma\left(c r^{*}\right)^{2}}{\lambda_{t} w(k)}\right)>2\left(\frac{\gamma\left(c r^{*}\right)^{2}}{\lambda_{t} w(k)}\right)>\frac{\left(\gamma c r^{*}\right)^{2}}{\lambda_{t} w(k)}$, then $e_{A, T}^{*}>e^{M}$.

Proof of Proposition (4): $\frac{d e_{A, T}^{*}}{d s_{t-1}}=\frac{d e^{M}}{d s_{t-1}}\left(1+2 e^{M} \lambda_{t} w(k)+2 r^{*} \gamma c\right)+$ $\lambda^{\prime}\left(s_{t-1}\right) w(k)\left(E^{2}-\left(e^{M}\right)^{2}+\left(\frac{\gamma c r^{*}}{\lambda_{t} w(k)}\right)^{2}\right)$, where $\frac{d e^{M}}{d s_{t-1}}=-\frac{c\left(1+r^{*}\right) \lambda^{\prime}\left(s_{t-1}\right)}{\left(\lambda_{t}\right)^{2} w(k)}$. It is straightforward that also $\frac{d e_{A, T}^{*}}{d s_{t-1}}<0$ and that $\frac{d e_{A, T}^{*}}{d s_{t-1}}>\frac{d e^{M}}{d s_{t-1}}$. Thus $\frac{d s_{t}}{d s_{t-1}}=$ $-\frac{1}{E} \frac{d e_{A, T}^{*}}{d s_{t-1}}>0$. By assuming that $E$ is high enough so that the most able worker will always choose to be educated even if no-one in the previous period was educated and since the agent $i$ with $e^{i}=0$ will never choose to be educated, then this implies that, as in Mountford, there must exist at least one steady state equilibrium for $s_{t}{ }^{6}$.

Proof of Proposition (5): $T^{* *}$ is always positive hence $e_{\pi}^{M}\left(\frac{r^{*} \gamma c}{2(1-\pi) E}\right)=$ $\left(\frac{r^{*} \gamma c \lambda_{t} w(k)}{2(1-\pi) E \bar{w}}\right)+\left(\frac{c}{\bar{w}}\right)\left(\frac{r^{*} \gamma c}{2(1-\pi) E}\right)+\left(\frac{\gamma\left(c r^{*}\right)^{2}}{2(1-\pi) E \bar{w}}\right)>\left(\frac{\gamma\left(c r^{*}\right)^{2}}{2(1-\pi) E \bar{w}}\right)>\frac{\left(\gamma c r^{*}\right)^{2}}{42(1-\pi) E \bar{w}}$ by the assumption $\gamma \leq 1$, then $\left(\frac{e_{\pi}^{M} r^{*}}{2(1-\pi) E}\right) \gamma c-\frac{\left(\gamma c r^{*}\right)^{2}}{4(1-\pi) \bar{w} E}>0$ and by $E>e_{\pi}^{M}$ then $\frac{\bar{w}\left(E^{2}-\left(e_{\pi}^{M}\right)^{2}\right)}{4 E(1-\pi)}$. Furthermore, the equation (2) and the maximin criterion ensure level of taxes lower enough to have educated agents.

Proof of Proposition (6): By $E>e^{M}$ and $2\left(e_{\pi}^{M} r^{*}\right) \gamma c=2\left(\frac{r^{*} \gamma c \lambda_{t} w(k)}{\bar{w}}\right)+$ $2\left(\frac{c}{\bar{w}}\right)\left(r^{*} \gamma c\right)+2\left(\frac{\gamma\left(c r^{*}\right)^{2}}{\bar{w}}\right)>2\left(\frac{\gamma\left(c r^{*}\right)^{2}}{\bar{w}}\right)>\frac{\left(\gamma c r^{*}\right)^{2}}{\bar{w}}$, then $e_{\pi, G}^{*}>e_{\pi}^{M}$. It is simple to how that $T^{* *}>T^{*}$.

Proof of Proposition (7): Following the proof of Mountford (see Mountford (1997) page 294, proposition 1), a sufficient condition for the existence of a positive level of brain drain (Brain Gain for the source economy) is that $\frac{d s_{t}}{d \pi}>0$ when $\pi=0$. The optimal level of $\pi$ will be given where $\frac{d s_{t}}{d \pi}=0$ although there

\footnotetext{
${ }^{6}$ See paragraph 2.1.5 of Mounftfors (1997) for furthermore details on the possible existence of multiple steady state.
} 
may exist other local maxima and minima. Differentiating the average proportion of educated people in the economy $s_{t}=\frac{(1-\pi) \int_{e_{\pi, G}^{*}}^{E} g e^{i} d e^{i}}{1-\pi\left(\int_{e_{\pi, G}^{*}}^{E} g e^{i} d e\right)}$ we solve $\frac{d s_{t}}{d \pi}=$ $\frac{\partial s_{t}}{\partial \pi}+\frac{\partial s_{t}}{\partial e_{\pi, G}^{*}} \frac{\partial e_{\pi, G}^{*}}{\partial \pi}$. The only difference respect the results of Mountford is given from $\frac{\partial e_{\pi, G}^{*}}{\partial \pi}=\left(\begin{array}{c}-\left(\lambda_{t} w(k)+c\left(1+r^{*}\right)\right)\left(1-2 \bar{w}\left(e_{\pi}^{M}\right)+2 r^{*} \gamma c\right)+ \\ +\left(E^{2}-\left(e_{\pi}^{M}\right)^{2}\right)\left(\lambda_{t} w(k)\right)^{2}+\left(\gamma c r^{*}\right)^{2}\end{array}\right) \frac{\left(w^{F}-\lambda_{t} w(k)\right)}{(\bar{w})^{2}}$. Setting $\pi=0$ and noting that $\int_{e_{\pi, G}^{*}}^{E} g\left(e^{i}\right) d e\left(1-\int_{e_{\pi, G}^{*}}^{E} g\left(e^{i}\right) d e^{i}\right)$ is at most a quarter, we have that $\frac{d s_{t}}{d \pi}>0$ when

$$
g\left(e_{A, T}^{*}\right)\left(\begin{array}{c}
\left(\lambda_{t} w(k)+c\left(1+r^{*}\right)\right) * \\
*\left(1-2 \lambda_{t} w(k)-2 c\left(1+(1-\gamma) r^{*}\right)\right)+ \\
-\left(E^{2}-\left(e_{\pi}^{M}\right)^{2}\right)\left(\lambda_{t} w(k)\right)^{2}-\left(\gamma c r^{*}\right)^{2}
\end{array}\right) \frac{\left(w^{F}-\lambda_{t} w(k)\right)}{\left(\lambda_{t} w(k)\right)^{2}}>\frac{1}{4} .
$$

Proof of Proposition (8): Comparing our condition to have a brain gain equation (14) and the result of Mountford $g\left(e_{A, T}^{*}\right)\left(\lambda_{t} w(k)+c\left(1+r^{*}\right)\right) \frac{\left(w^{F}-\lambda_{t} w(k)\right)}{\left(\lambda_{t} w(k)\right)^{2}}>$ $\frac{1}{4}$ and noting that $-\left(E^{2}-\left(e_{\pi}^{M}\right)^{2}\right)(\bar{w})^{2}-\left(\gamma c r^{*}\right)^{2}<0$ and $1-2 \lambda_{t} w(k)-2 c(1+$ $\left.(1-\gamma) r^{*}\right)<1$, it is straightforward that the LHS of the condition with government is lower than the case without government (Mountford case).

\section{Appendix B}

\section{Autarchic case}

The maximization program of the government is

$$
\underset{T}{\operatorname{Max} b}
$$

where, by equations (2) and (3)

$$
\begin{gathered}
b=-\frac{1}{2}\left(\lambda_{t} w(k) e^{M}+2 T-2 \gamma c-\gamma c r^{*}\right)+ \\
+\frac{1}{2}\left[\begin{array}{c}
\left(\lambda_{t} w(k) e^{M}+2 T-2 \gamma c-\gamma c r^{*}\right)^{2}+ \\
+4\left(\lambda_{t} w(k)\left(E-e^{M}\right)-T+\gamma c\left(1+r^{*}\right)\right)(T-\gamma c)
\end{array}\right]^{\frac{1}{2}}
\end{gathered}
$$

The F.O.C. is

$$
\begin{gathered}
-2+\frac{1}{2}\left[\begin{array}{c}
\left(\lambda_{t} w(k) e^{M}+2 T-2 \gamma c-\gamma c r^{*}\right)^{2}+ \\
+4\left(\lambda_{t} w(k)\left(E-e^{M}\right)-T+\gamma c\left(1+r^{*}\right)\right)(T-\gamma c)
\end{array}\right]^{-\frac{1}{2}} * \\
*\left[\begin{array}{c}
4\left(\lambda_{t} w(k) e^{M}+2 T-2 \gamma c-\gamma c r^{*}\right)-4(T-\gamma c)+ \\
+4\left(\lambda_{t} w(k)\left(E-e^{M}\right)-T+\gamma c\left(1+r^{*}\right)\right)
\end{array}\right]=0
\end{gathered}
$$


or, after algebraically computation

$$
\begin{aligned}
& \lambda_{t} w(k) e^{M}+2 T-2 \gamma c-\gamma c r^{*}-T+\gamma c+\lambda_{t} w(k)(E)-\lambda_{t} w(k)\left(e^{M}\right)-T+\gamma c+\gamma c r^{*} \\
= & {\left[\left(\lambda_{t} w(k) e^{M}+2 T-2 \gamma c-\gamma c r^{*}\right)^{2}+4\left(\lambda_{t} w(k)\left(E-e^{M}\right)-T+\gamma c\left(1+r^{*}\right)\right)(T-\gamma c)\right]^{\frac{1}{2}} }
\end{aligned}
$$

or

$$
\left(\lambda_{t} w(k) E\right)^{2}=\left(\lambda_{t} w(k) e^{M}+2 T-2 \gamma c-\gamma c r^{*}\right)^{2}+4\left(\lambda_{t} w(k)\left(E-e^{M}\right)-T+\gamma c\left(1+r^{*}\right)\right)(T-\gamma c)
$$

or

$$
\begin{aligned}
\left(\lambda_{t} w(k) E\right)^{2}= & \left(\lambda_{t} w(k) e^{M}-\gamma c r^{*}\right)^{2}+(2(T-\gamma c))^{2}+4\left(\lambda_{t} w(k) e^{M}-\gamma c r^{*}\right)(T-\gamma c) \\
& \left.-4\left(\lambda_{t} w(k) e^{M}-\gamma c r^{*}\right)\right)(T-\gamma c)+4\left(\lambda_{t} w(k) E\right)(T-\gamma c)-4(T-\gamma c)^{2}
\end{aligned}
$$

or

$$
\left(\lambda_{t} w(k) E\right)^{2}=\left(\lambda_{t} w(k) e^{M}-\gamma c r^{*}\right)^{2}+4\left(\lambda_{t} w(k) E\right)(T-\gamma c)
$$

finally, the optimal value of taxation is

$$
T^{*}=\frac{\lambda_{t} w(k)\left(E^{2}-\left(e^{M}\right)^{2}\right)}{4 E}+\gamma c+\left(\frac{e^{M} r^{*}}{2 E}\right) \gamma c-\frac{\left(\gamma c r^{*}\right)^{2}}{4 \lambda_{t} w(k) E}>0 .
$$

\section{Mobility case}

The maximization program of the government is

$$
\underset{T}{\operatorname{Max} b}
$$

where, by equations (9) and (10)

$$
\begin{gathered}
b=-\frac{\left(\bar{w} e_{\pi}^{M}+2(1-\pi) T-2 \gamma c-\gamma c r^{*}\right)}{2}+ \\
+\frac{1}{2}\left[\begin{array}{c}
\left(\bar{w} e_{\pi}^{M}+2(1-\pi) T-2 \gamma c-\gamma c r^{*}\right)^{2}+ \\
+4\left(\bar{w}\left(E-e_{\pi}^{M}\right)-(1-\pi) T+\gamma c\left(1+r^{*}\right)\right)((1-\pi) T-\gamma c)
\end{array}\right]^{\frac{1}{2}}
\end{gathered}
$$

The F.O.C. is

$$
\underset{T}{\operatorname{Max} b}
$$

$$
\begin{aligned}
& \frac{1}{2}\left[\begin{array}{c}
\left(\bar{w} e_{\pi}^{M}+2(1-\pi) T-2 \gamma c-\gamma c r^{*}\right)^{2}+ \\
+4\left(\bar{w}\left(E-e_{\pi}^{M}\right)-(1-\pi) T+\gamma c\left(1+r^{*}\right)\right)((1-\pi) T-\gamma c)
\end{array}\right]^{-\frac{1}{2}} * \\
* & {\left[\begin{array}{c}
4(1-\pi)\left(\bar{w} e_{\pi}^{M}+2(1-\pi) T-2 \gamma c-\gamma c r^{*}\right)+ \\
-4(1-\pi)((1-\pi) T-\gamma c)+ \\
+4(1-\pi)\left(\bar{w}\left(E-e_{\pi}^{M}\right)-(1-\pi) T+\gamma c\left(1+r^{*}\right)\right)
\end{array}\right]-2(1-\pi)=0 }
\end{aligned}
$$


or, after algebraically computation

$$
\begin{gathered}
-1+\left[\begin{array}{c}
\left(\bar{w} e_{\pi}^{M}+2(1-\pi) T-2 \gamma c-\gamma c r^{*}\right)^{2}+ \\
+4\left(\bar{w}\left(E-e_{\pi}^{M}\right)-(1-\pi) T+\gamma c\left(1+r^{*}\right)\right)((1-\pi) T-\gamma c)
\end{array}\right]^{-\frac{1}{2}} * \\
\bar{w} e_{\pi}^{M}+2(1-\pi) T-2 \gamma c-\gamma c r^{*}+ \\
*\left[\begin{array}{c}
\left.(1-\pi)\left(E-e_{\pi}^{M}\right)-(1-\pi) T+\gamma c\left(1+r^{*}\right)\right) \\
-((1-\pi) T-\gamma c)+(\bar{w}(E)=0
\end{array}\right.
\end{gathered}
$$

or

$$
\begin{aligned}
& \bar{w} e_{\pi}^{M}+2(1-\pi) T-2 \gamma c-\gamma c r^{*}-(1-\pi) T+ \\
& +\gamma c+\bar{w}(E)-\bar{w}\left(e_{\pi}^{M}\right)-(1-\pi) T+\gamma c+\gamma c r^{*} \\
= & {\left[\begin{array}{c}
\left(\bar{w} e_{\pi}^{M}+2(1-\pi) T-2 \gamma c-\gamma c r^{*}\right)^{2}+ \\
+4\left(\bar{w}\left(E-e_{\pi}^{M}\right)-(1-\pi) T+\gamma c\left(1+r^{*}\right)\right)((1-\pi) T-\gamma c)
\end{array}\right]^{\frac{1}{2}} }
\end{aligned}
$$

or

$$
\begin{aligned}
& (\bar{w} E)^{2}=\left(\bar{w} e_{\pi}^{M}-\gamma c r^{*}\right)^{2}+(2((1-\pi) T-\gamma c))^{2}+4\left(\bar{w} e_{\pi}^{M}-\gamma c r^{*}\right)((1-\pi) T-\gamma c) \\
& -4\left(\bar{w} e_{\pi}^{M}-\gamma c r^{*}\right)((1-\pi) T-\gamma c)+4(\bar{w} E)((1-\pi) T-\gamma c)-4((1-\pi) T-\gamma c)^{2}
\end{aligned}
$$

or

$$
(\bar{w} E)^{2}=\left(\bar{w} e_{\pi}^{M}-\gamma c r^{*}\right)^{2}+4(\bar{w} E)((1-\pi) T-\gamma c)
$$

finally, the optimal value of taxation is

$$
T^{* *}=\frac{\bar{w}\left(E^{2}-\left(e_{\pi}^{M}\right)^{2}\right)}{4 E(1-\pi)}+\frac{\gamma c}{(1-\pi)}+\left(\frac{e_{\pi}^{M} r^{*}}{2(1-\pi) E}\right) \gamma c-\frac{\left(\gamma c r^{*}\right)^{2}}{4(1-\pi) \bar{w} E}>T^{*}
$$

